\title{
Setting up a service for a faecal immunochemical test for haemoglobin (FIT): a review of considerations, challenges and constraints
}

\author{
Ian M Godber, ${ }^{1}$ Sally C Benton, ${ }^{2,3}$ Callum G Fraser ${ }^{4}$
}

\begin{abstract}
'Department of Clinical Biochemistry, NHS Lanarkshire, Monklands Hospital, Airdrie, Lanarkshire, UK

${ }^{2}$ Berkshire and Surrey Pathology Services, Royal Surrey County Hospital, Guildford, UK

${ }^{3}$ Bowel Cancer Screening Hub, Guildford, UK

${ }^{4}$ Centre for Research into Cancer Prevention and

Screening, University of Dundee, Ninewells Hospital and Medical School, Dundee, UK
\end{abstract}

Correspondence to Dr lan M Godber, Department of Clinical Biochemistry, Monklands Hospital, Airdrie ML60JS, UK; lan.Godber@ lanarkshire.scot.nhs.uk

Received 11 July 2018 Revised 28 August 2018 Accepted 6 September 2018 Published Online First 1 October 2018

\begin{abstract}
Quantitative faecal immunochemical tests for haemoglobin (FIT) have now been advocated by the National Institute for Care and Health Excellence (NICE: DG30) to assist in the triage of patients presenting with symptoms that suggest a low risk of colorectal (bowel) cancer. The evidence is that FIT provides a good rule out test for significant bowel disease. However, a small number of cases will be missed, and robust safetynetting procedures are required to follow up some FIT-negative patients. A range of diagnostic pathways are possible, and there is no best approach at present. Introduction of FIT requires careful consideration of the logistics of supply of devices and information to requesting sites and of transport to the laboratory. $A$ number of FIT analytical systems are available. Three are documented as appropriate for use in assessment of patients with symptoms. However, preanalytical, analytical and postanalytical challenges remain. The methods have different specimen collection devices. The methods use polyclonal antibodies and there is no primary reference material or method to which FIT methods are standardised. Third-party internal quality control is lacking, and external quality assessment schemes have many difficulties in providing appropriate materials. Reporting of results should be done using $\mu \mathrm{g} \mathrm{Hb} / \mathrm{g}$ faeces units and with knowledge of the limit of detection and limit of quantitation of the analytical system used. FIT can be used successfully in an agreed diagnostic pathway, along with other clinical and laboratory information: this requires a multidisciplinary approach, providing opportunities for professionals in laboratory medicine involvement.
\end{abstract}

\section{BACKGROUND}

The National Institute for Health and Care Excellence (NICE) published guidance on Suspected Cancer: Recognition and Referral (NG12) in 2015. ${ }^{1}$ This guideline was aimed at identifying people with symptoms that could be caused by cancer, with recommendations on the investigations to aid referral. A controversial statement in this document, relating to investigation of lower bowel symptoms, was the recommendation that testing for occult blood in faeces should be undertaken in cases where there was a low risk of colorectal (bowel) cancer (CRC). This was universally interpreted as advocating the use of traditional guaiac-based faecal occult blood tests (gFOBT), the evidence based on a small number of studies; faecal immunochemical tests for haemoglobin (FIT) were not widely available in routine laboratory use at the time. The qualitative gFOBT has many documented disadvantages, ${ }^{2}$ was not previously recommended for use in patients with symptoms and was considered obsolete; in consequence, gFOBT had been withdrawn from the repertoire of many laboratories and their use in other settings such as wards and clinics was much discouraged. Laboratories were then faced with the dilemma of how to comply with the recommendations put forward within the NG12 guideline, either reintroduce gFOBT or investigate the possibility of introducing the reportedly superior FIT. In reality, a large number of laboratories offered neither and, in consequence, general practitioners (GPs) were unable to comply with the NG12 guidance. This review aims to address some of the issues likely to be encountered by laboratories and the factors that are required to be taken into account when introducing a service providing FIT to aid in the assessment of patients presenting with lower bowel symptoms, it is concerned only with patients presenting with symptoms and not with the asymptomatic population-based bowel cancer screening, already carried out in national programmes in operation within the United Kingdom.

\section{Evidence for the use of FIT in assessment of the symptomatic patients}

Following the criticism of the 2015 NG12 recommendations and in response to the questions being asked by professionals in laboratory medicine in the UK, NICE set up a Diagnostic Assessment Group (DAG) to review the evidence for the use of quantitative FIT in assessment of patients with low-risk symptoms and also commissioned a systematic review of the work done on this topic. There are a small number of studies on assessment of patients with symptoms using qualitative FIT and other tests such as faecal calprotectin, ${ }^{3}$ but these were objectively excluded from the DAG review. The review group report was published (DG30) in $2017^{4}$ and concluded that there was sufficient evidence to advocate triage of symptomatic patients using faecal haemoglobin concentration ( $\mathrm{f}-\mathrm{Hb}$ ) at a cut-off of 10 $\mu \mathrm{g} \mathrm{Hb} / \mathrm{g}$ faeces and that this had the potential to correctly rule out CRC and avoid colonoscopy in $75 \%-80 \%$ of cases. The evidence reviewed in order to propose this cut-off of $10 \mu \mathrm{g} \mathrm{Hb} / \mathrm{g}$ faeces is documented in the systematic review, which followed the publication of the DG30 guideline. ${ }^{5}$ The publication of DG30 led to an update of the 2015 NG12 guideline that now recommends that the DG30 
guidance should be followed for patients at low risk of CRC using FIT; the references to gFOBT have been eliminated. ${ }^{2}$ Since the systematic review of Westwood et $\mathrm{al}^{5}$ there have been a small number of further publications on this topic and these have been reviewed recently ${ }^{67}$

Added to this, when referral is made into secondary care following a positive FIT result, the quantitative result may allow those with elevated $\mathrm{f}-\mathrm{Hb}$ more in keeping with malignancy to be triaged effectively ${ }^{8}$ since $\mathrm{f}-\mathrm{Hb}$ is directly related to the severity of colorectal disease, with more significant bowel diseases (SBDs) being associated with higher $\mathrm{f}-\mathrm{Hb} .{ }^{9}$ Indeed, it has been suggested that the $\mathrm{f}-\mathrm{Hb}$ could be used to prioritise further investigation, those with higher $\mathrm{f}-\mathrm{Hb}$ being further investigated sooner. ${ }^{34}$ These factors therefore allow FIT to be built into a diagnostic pathway that can be used for effective referral and triage of symptomatic patients.

All studies included in the DG30 guideline demonstrate very similar outcomes: that FIT has a high negative predictive value and, as such, is good as a rule out test for SBD (SBD =CRC + higher-risk adenoma (HRA)+inflammatory bowel disease (IBD). However, it is important to recognise that it is reported in a number of the published studies that CRC (and HRA and IBD) would have been missed in a very small proportion of the population tested at the cut-off of $10 \mu \mathrm{g} \mathrm{Hb} / \mathrm{g}$ faeces recommended in DG30 and the 2017 NG12 guidance, and this is important to consider when working with primary care colleagues to implement FIT; it is imperative to ensure that GP and other healthcare professionals are aware of this risk so that safety-netting can be put in place to appropriately follow up patients with $\mathrm{f}-\mathrm{Hb}$ below the $10 \mu \mathrm{g} \mathrm{Hb} / \mathrm{g}$ faeces cut-off who continue to be symptomatic. Such safety-netting must be built into any clinical pathway prior to implementation and might advantageously include triaging patients with iron deficient anaemia or ongoing diarrhoea to colonoscopy, irrespective of the FIT result.

The evidence used to support the DG30 guidance includes a total of 17 publications from 10 studies. One study was only published in abstract form at the time of the guideline publication but has now been published as a full paper; interestingly, only some of the patients studied were symptomatic and many were in surveillance following previous disease. ${ }^{10}$ The total numbers of patients across all studies was 5062; 4091 (OC-Sensor, Eiken Chemical, Tokyo, Japan), 484 (HM-JACKarc, Kyowa Medex, Tokyo, Japan) and 487 (FOB Gold, Sentinel Diagnostics, Milan, Italy). While the NICE DG30 guideline is for patients at low risk for CRC, all studies used in evidence included all patients who were referred to colonoscopy and, dditionally, all studies only did a FIT on patients already referred for colonoscopy, presumably based on clinical symptoms and the results of examination and possibly other investigations. To date, there are limited studies investigating the use of FIT for patients at low risk, and further evidence is required in this population. A study carried out in 3462 indiviuals presenting with low risk or 'non-alarm' symptoms in Denmark concluded that FIT may also be used as a supplementary diagnostic test in a pathway for the diagnosis of CRC and other SBDs in this population. ${ }^{11}$

Building on the evidence collated for NICE DG30, it is considered that further research is required to consider the influence of a patient's sex, age and medication on the application of partitioned cut-off for FIT. It has been established that $\mathrm{f}-\mathrm{Hb}$ is higher in men than in women and increases with age and, importantly, these relationships vary from country to country. ${ }^{12} 13$ Moreover, $\mathrm{f}-\mathrm{Hb}$ increases as deprivation increases. ${ }^{13} 14$ One potential solution that takes these and other relevant variables into consideration in combination with the $\mathrm{f}-\mathrm{Hb}$ concentration are risk-scoring models such as the very simple Score Card, ${ }^{15}$ the very complex COLONPREDICT mode $^{16}$ and the FAST score, which simply takes $\mathrm{f}-\mathrm{Hb}$, age and sex into account. ${ }^{17}$ However, their use in routine practice has yet to be evaluated in depth, but this sort of approach might simplify the interpretation of FIT results among GP considering making a referral to secondary care, particularly since the scores could be reported using the functionality available in most laboratory information systems, perhaps along with interpretative guidance.

In terms of methodology, there are four quantitative FIT systems currently available on the UK market. All are used in CRC screening programmes across the world. Only three of these FIT systems were recommend in the DG30 guidance, and the reason for this is that they had to have data on assessment of patients with symptoms for acceptance into the guidance and one system (NS-Prime, Alfresa, Japan) did not.

\section{FIT in a diagnostic referral pathway}

Since it is proposed that the use of FIT can aid a decision to refer made using a pathway for the investigation of suspected CRC and other SBD, consideration should be made when developing such a pathway as to who can request the test and by whom the test results are interpreted. In those centres in the UK who have implemented the test, current practice varies considerably, with results either going back to the GP or returned to secondary care gastroenterology or gastric surgery, depending on the referral pathway being used. The reasons for this variation in practice are considered below and are dependent on the clinical question being addressed and the key drivers for the introduction of FIT. At present, there is no single best approach that can be recommended for ubiquitous adoption.

There have been increasing referrals for colonoscopy from primary care, putting the service under considerable pressure, particularly following the introduction of bowel screening programmes in all four UK nations and with the introduction of local, regional and national publicity regarding the importance of seeking healthcare when lower bowel symptoms are experienced. As pointed out in the NICE: DG30 guideline, FIT has the potential to correctly rule out CRC and other SBD and avoid colonoscopy. Thus, measurement of $\mathrm{f}-\mathrm{Hb}$ is potentially a useful tool to avoid unnecessary invasive procedures that themselves carry an inherent risk of bleeding, perforation and possible death. Although the risk is small for diagnostic colonoscopy, it increases markedly when combined with therapeutic procedures carried out at the same time including polypectomy and, in four recent studies, overall perforation rates ranged from $0.03 \%$ to $0.09 \% .^{18}$

One example of a referral pathway is illustrated in figure 1 . Research studies give evidence that FIT, as an adjunct to clinical information and full blood count, can provide a very useful tool to assist with the triage of patients presenting in primary care with lower abdominal symptoms. An analysis of the three studies carried out to date using FIT in symptomatic patients in Scotland suggested that, within this cohort, there was potential to have avoided a referral and subsequent invasive investigation in $82.2 \%$ patients without SBD $^{19}$ if a pathway similar to this is followed. However, it is vital to note that FIT are not $100 \%$ sensitive and rely on the pathological lesion to be bleeding at the time of faecal sampling and require the faeces to transit the colon before any haemoglobin present is degraded; in the 1514 cases reviewed by Quyn et al ${ }^{19}$ three cases of CRC would have been missed using $\mathrm{f}-\mathrm{Hb}$ with a $10 \mu \mathrm{g} \mathrm{Hb} / \mathrm{g}$ faeces cut-off, all three cases had $\mathrm{f}-\mathrm{Hb}$ above the limit of detection of the FIT 


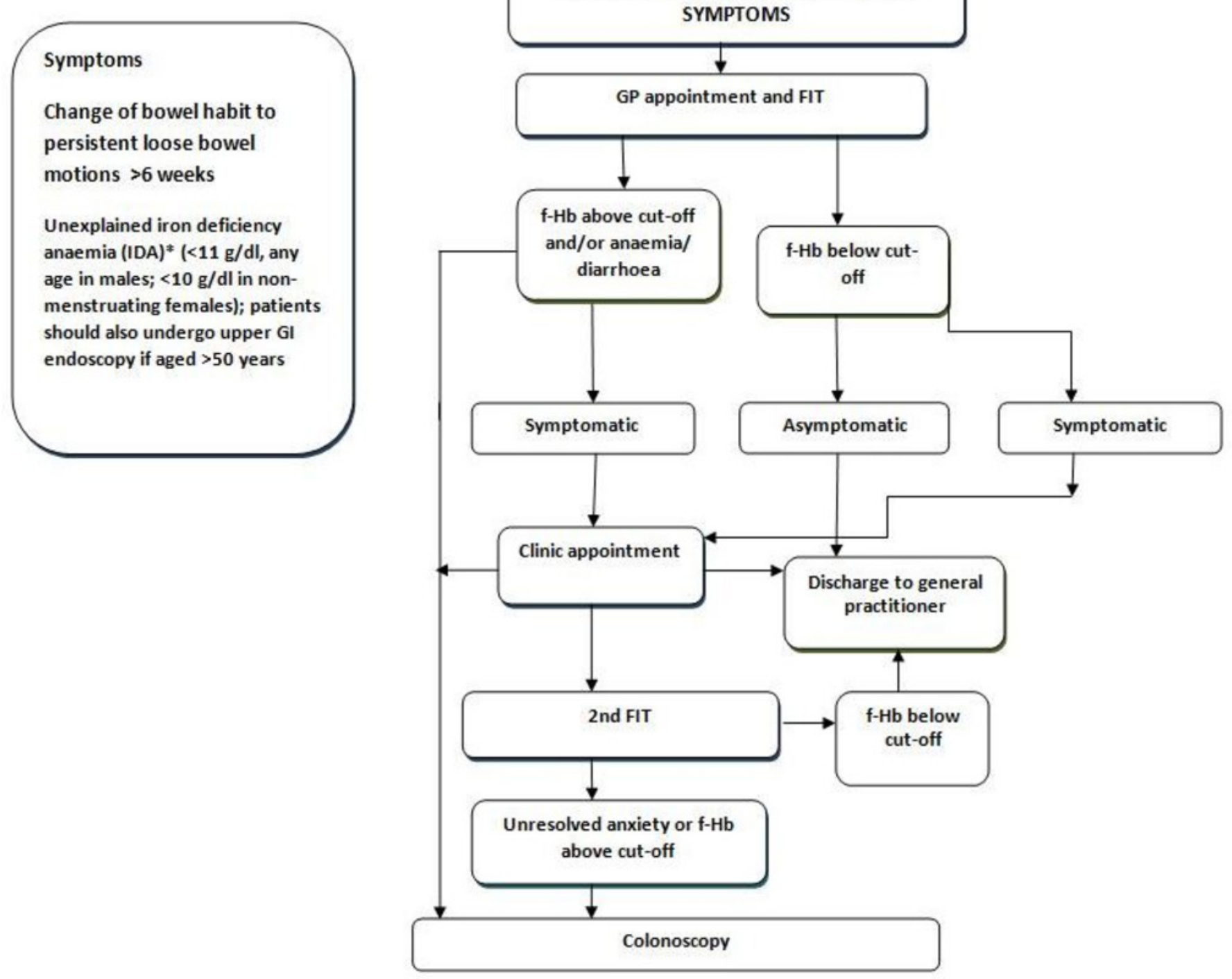

Figure 1 Example of a referral pathway for colonoscopy incorporating FIT. f-Hb, faecal haemoglobin concentration; FIT, faecal immunochemical tests for haemoglobin; GP, general practitioner.

system used but $\mathrm{f}-\mathrm{Hb}$ below the recommended $10 \mu \mathrm{g} \mathrm{Hb} / \mathrm{g}$ faeces cut-off. This has confirmed that a robust safety-netting procedure should be built into any clinical pathway for patients with symptoms. Watching and waiting could be supplemented by referral to gastroenterology or other appropriate clinic in secondary care, particularly if there are ongoing symptoms such as recurrent diarrhoea and/or iron-deficient anaemia, although patients with such serious symptoms should probably be referred for further investigation directly, irrespective of the $\mathrm{f}-\mathrm{Hb}$ found, since these symptoms have higher positive predictive value for SBD than other low risk symptoms documented in the NICE NG12 guidelines. Some have suggested that a repeat FIT could be done, particularly if there are ongoing problems, but there are no data on this approach to date to our knowledge; studies on outcomes using this strategy would be of much interest.

In order to have an impact on the number of referrals to secondary care, the FIT result should be available to the referring clinician to enable an informed decision to be made. If this is the GP, then those implementing the clinical pathway need to consider the education and training of their colleagues in primary care. Supplementary information could also be available in the form of interpretive comments on reports that accompany the results. Moreover, regular newsletters giving, for example, number of requests, use by different GP, clinical outcomes, audit information and synopses of interesting cases encountered, publications or new relevant guidelines might be of value, especially if web based. This approach has the advantage that all patients with symptoms receive a FIT and the $\mathrm{f}-\mathrm{Hb}$ can, along with the impression of the GP and the results of other tests, particularly the full blood count, result in an objective decision to refer or not refer, saving secondary care from deciding on the need for colonoscopy or a secondary care clinic appointment. The disadvantage is the FIT workload would be high since patients presenting with lower bowel symptoms are very commonly seen in primary care.

An alternative implementation strategy might be to make FIT only available as part of the referral mechanism to secondary care. Here, the GP would make a referral to secondary care for a colonoscopy or outpatient review with clinical information as required by local practice and IT systems but would also request 
a FIT. The result of the FIT would be returned to the secondary care clinician who could use it to decide on the relevance of the referral. Options could include fast tracking the patient straight to colonoscopy, review in an outpatient clinic or discharge back to the care of the GP, perhaps with guidance on further follow-up. The advantage of this approach is the smaller pool of clinical staff required to be trained to make an informed decision based on the $\mathrm{f}-\mathrm{Hb}$ result and therefore have the opportunity to gain more experience in interpretation and comparing this with outcome data. The disadvantage in this approach is that this will not reduce the number of patients referred to secondary care; however, it is still likely to impact the number of colonoscopies carried out.

When planning an implementation strategy for FIT, the other key factors to consider are the logistics of supply of FIT kits and specimen transport. The most widely used methods for FIT in use in the UK use their own individual proprietary specimen collection devices. These are certainly preferred to the traditional faecal collection pots since there is evidence that $\mathrm{f}-\mathrm{Hb}$ decreases rapidly with time when not protected by collection into the preservative buffer in the devices. ${ }^{2021}$ In order for the requestor to be able to give these devices to the patient, adequate supplies must be distributed to each requesting location, along with information (preferably mainly pictorial) on how to correctly collect the faecal sample into the device and a return envelope or container. This can be a labour-intensive process and one that requires continuous management and monitoring including keeping a record of the expiry dates of collection devices. The devices are approved for sending through the mail in the UK, and posting the sample to the laboratory may provide a patient-friendly alternative to returning the sample to the GP for onward transmission to the laboratory.

\section{ANALYTICAL CONSIDERATIONS}

As well as the procedural considerations that must be appraised and the challenges solved when setting up a service for assessment of symptomatic patients using FIT, there are many analytical considerations which must also be taken into account.

FIT use polyclonal antibodies raised against the globin moiety of the $\mathrm{Hb}$ molecule. These antibodies are specific to human globin so, unlike the traditional gFOBT, they are not prone to interference from any haemoglobin derived from the diet or from any substance that interferes in the redox reaction of guaiac, such as peroxidases. Moreover, a recent systematic review has shown that cessation of oral anticoagulants, aspirin and other non-steroidal anti-inflammatory drugs is not required. ${ }^{22}$ There are a large number of FIT on the market; many of these are qualitative tests with very little evidence to support their use in any clinical setting. There are an increasing number of quantitative FIT analytical approaches on the market, mainly designed for CRC screening, ${ }^{23}$ and four of these were subject to an independent analytical evaluation that ratified their potential use in the diagnosis of SBD. ${ }^{24}$

While there are a large number of FIT methods available in the UK, both qualitative and quantitative, the latter are generally used, as recommended in DG30. Faecal samples for quantitative FIT are collected at home by the patient. A small mass of faeces $(2 \mathrm{mg}$ or $10 \mathrm{mg})$ is loaded on to a grooved or dimpled probe attached to the cap of the specimen collection device and then this is reinserted into the body of the device that contains a preservative buffer that stabilises any $\mathrm{Hb}$ present. Excess faeces on the probe is removed by an internal collar leaving a consistent mass of faeces in the buffer. ${ }^{25}$
There are a number of preanalytical and analytical challenges with FIT. There is no harmonisation of the sample collection devices. All have different designs of grooves or dimples ${ }^{26}$ in which to collect the sample and, due to the heterogeneity of faecal samples, potentially the amount of $\mathrm{f}-\mathrm{Hb}$ collected will vary dependant from where in the faeces the sample is collected. Manufacturers' recommendations on how to collect faeces using their devices varies, with some recommending sticking the sample probe into the faeces a number of times and others advocating swiping across the surface of the faeces. Faeces has variable consistency, and there are no studies to date that investigate the impact of this on $\mathrm{f}-\mathrm{Hb}$ results. Analytically, there is no standardisation of FIT. There is currently no primary reference material or method to which FIT methods are standardised. Work is being done to address these problems internationally through an International Federation of Clinical Chemistry Scientific Division Working Group in which the four major FIT manufacturers participate, and a reference material is in the process of being investigated. A small study carried out recently has demonstrated that the use of polyclonal antibodies appears to limit problems in antibody detection of most haemoglobin variants; those variants with a missing alpha or beta globin chain are under-recovered with FIT and hence patients with such $\mathrm{Hb}$ variants are at risk of falsely low or negative $\mathrm{f}-\mathrm{Hb}$ results. ${ }^{27}$

There are no established third-party internal quality control (IQC) materials currently available in the UK; however, materials have recently been made available elsewhere in Europe. If introducing an IQC protocol, factors considered in a previous review in this journal should be taken into account. ${ }^{28}$ It has also been challenging for external quality assessment scheme (EQAS) organisers to develop meaningful EQAS to assess FIT analytical performance; EQAS that send out faecal like matrices spiked with $\mathrm{Hb}$ exhibit large variation in results from the participant laboratories, and it is difficult to ascertain whether these differences are preanalytical or analytical. Conversely, those schemes that send out lyophilised aqueous samples of $\mathrm{Hb}$, while providing a means of assessing comparative analytical bias, are not conforming to the traditional requirements of a commutable EQAS sample being in a matrix that reflects from sample patients.

Harmonisation of reporting units is also an issue, and while the international guidelines ${ }^{29}$ recommended that FIT be reported in $\mu \mathrm{g} \mathrm{Hb} / \mathrm{g}$ faeces rather than $\mathrm{ng} \mathrm{Hb} / \mathrm{mL}$ buffer, this still requires manufacturers to have a conversion in their analysers which is dependent on the both the mass of faeces collected and the volume of buffer in the specimen collection device in order to report comparable results.

DG30 guidance recommends a cut-off of $10 \mu \mathrm{g} \mathrm{Hb} / \mathrm{g}$ faeces for referral of patients who present with symptoms in primary care. All three FIT systems recommended have performance characteristics that allow reporting of a quantitative $\mathrm{f}-\mathrm{Hb}$ result, since this cut-off is equal to or higher than the documented limits of quantitation (LoQ), defined as the lowest concentration at which the performance meets the defined analytical performance specifications of the assay. ${ }^{30}$ Some studies have reported at lower f-Hb cut-offs than the LoQ. It is important that a local analytical verification of a FIT system is carried out, including confirmation of the LoQ, before reporting $\mathrm{f}-\mathrm{Hb}$ lower than the $10 \mu \mathrm{g}$ $\mathrm{Hb} / \mathrm{g}$ faeces cut-off and any publications including data below $10 \mu \mathrm{g} \mathrm{Hb} / \mathrm{g}$ faeces need to be evidenced to confirm the validity of the cut-off applied. It may be that f-Hb results lower than the LoQ but above the limit of detection, the highest measured result likely to be observed (typically at 95\% certainty) for a sample containing no $\mathrm{f}-\mathrm{Hb}$ could be reported as $\mathrm{f}-\mathrm{Hb}$ detected 
but without a numerical result. Detailed recommendations for reporting $\mathrm{f}-\mathrm{Hb}$ data at low concentrations are made in a recent opinion paper along with the interim proposal that the analytical performance specification for $\mathrm{f}-\mathrm{Hb}$ should be that imprecision (and measurement uncertainty) be based on the current state of the art, that $\mathrm{CV}$ be $<10 \%$. $^{31}$

\section{CONCLUSIONS}

The introduction of FIT into routine medical laboratories is a rapidly developing area of interest, and new evidence is being regularly published, adding to the knowledge base. There are a significant number of factors that need to be taken into account when setting up such a service, including preanalytical, analytical and postanalytical aspects; however, using FIT as a tool within an agreed diagnostic pathway, along with other clinical and laboratory information, is vital for a successful implementation. Such an approach requires multidiscipline coordination and cooperation and is an opportunity for professionals in laboratory medicine to build productive links with a broad spectrum of their clinical colleagues.

\section{Handling editor Tahir S Pillay.}

Contributors All authors contributed equally for the production of this work.

Funding The authors have not declared a specific grant for this research from any funding agency in the public, commercial or not-for-profit sectors.

Competing interests CGF undertakes consultancy with Kyowa Medex Co, Ltd, Tokyo, Japan, and has received support to attend conferences from Alpha labs Ltd, Eastleigh, Hants, UK.

Patient consent Not required.

Provenance and peer review Commissioned; externally peer reviewed.

\section{REFERENCES}

1. National Institute for Health and Care Excellence, 2015. NICE guideline 12. Suspected cancer: recognition and referral. Available from: https://www.nice.org.uk/guideance/ ng12

2. Fraser CG. Faecal occult blood tests--eliminate, enhance or update? Ann Clin Biochem 2008:45(Pt 2):117-21.

3. Steele RJC, Fraser CG, Olsson L. Faecal immunochemical tests (FIT) for haemoglobin for timely assessment of patients with symptoms of colorectal disease. Timely diagnosis of colorectal cancer. Cham: Springer, 2018.

4. NICE, 2017. Diagnostics guidance DG30. Quantitative faecal immunochemical tests to guide referral for colorectal cancer in primary care. Available from: https://www.nice. org.uk/guidance/dg30

5. Westwood M, Lang S, Armstrong N, et al. Faecal immunochemical tests (FIT) can help to rule out colorectal cancer in patients presenting in primary care with lower abdominal symptoms: a systematic review conducted to inform new NICE DG30 diagnostic guidance. BMC Med 2017;15:189.

6. Fraser CG. Faecal immunochemical tests (FIT) in the assessment of patients presenting with lower bowel symptoms: Concepts and challenges. Surgeon 2018;16:302-8.

7. Spiteri N, Skaife P. Get FIT for the new year: a review of the role of faecal immunochemical test for haemoglobin in patients with symptoms of colorectal disease. J Lab Precis Med 2018:3:52

8. Godber IM, Todd LM, Fraser CG, et al. Use of a faecal immunochemical test for haemoglobin can aid in the investigation of patients with lower abdominal symptoms. Clin Chem Lab Med 2016;54:595-602.
9. Digby J, Fraser CG, Carey FA, et al. Faecal haemoglobin concentration is related to severity of colorectal neoplasia. J Clin Pathol 2013;66:415-9.

10. Auge JM, Rodriguez C, Espanyol 0, et al. An evaluation of the SENTiFIT 270 analyser for quantitation of faecal haemoglobin in the investigation of patients with suspected colorectal cancer. Clin Chem Lab Med 2018;56:625-33.

11. Juul JS, Hornung N, Andersen B, et al. The value of using the faecal immunochemical test in general practice on patients presenting with non-alarm symptoms of colorectal cancer. Br J Cancer 2018:119:471-9.

12. Fraser CG, Rubeca T, Rapi $\mathrm{S}$, et al. Faecal haemoglobin concentrations vary with sex and age, but data are not transferable across geography for colorectal cancer screening. Clin Chem Lab Med 2014;52:1211-6.

13. Symonds EL, Osborne JM, Cole SR, et al. Factors affecting faecal immunochemical test positive rates: demographic, pathological, behavioural and environmental variables. J Med Screen 2015;22:187-93.

14. Digby J, McDonald PJ, Strachan JA, et al. Deprivation and faecal haemoglobin: implications for bowel cancer screening. J Med Screen 2014;21:95-7.

15. Rodríguez-Alonso L, Rodríguez-Moranta F, Ruiz-Cerulla A, et al. An urgent referral strategy for symptomatic patients with suspected colorectal cancer based on a quantitative immunochemical faecal occult blood test. Dig Liver Dis 2015;47:797-804

16. Cubiella J, Vega P, Salve M, et al. Development and external validation of a faecal immunochemical test-based prediction model for colorectal cancer detection in symptomatic patients. BMC Med 2016;14:128

17. Cubiella J, Digby J, Rodríguez-Alonso L, et al. The fecal hemoglobin concentration, age and sex test score: Development and external validation of a simple prediction tool for colorectal cancer detection in symptomatic patients. Int J Cancer 2017;140:2201-11.

18. Rees CJ, Thomas Gibson S, Rutter MD, et al. UK key performance indicators and quality assurance standards for colonoscopy. Gut 2016;65:1923-9.

19. Quyn AJ, Steele RJ, Digby J, et al. Application of NICE guideline NG12 to the initial assessment of patients with lower gastrointestinal symptoms: not FIT for purpose? Ann Clin Biochem 2018;55:69-76.

20. Brown LF, Fraser CG. Effect of delay in sampling on haemoglobin determined by faecal immunochemical tests. Ann Clin Biochem 2008;45(Pt 6):604-5.

21. Mellen $S$, de Ferrars $M$, Chapman $C$, et al. Evaluation of sample stability for a quantitative faecal immunochemical test and comparison of two sample collection approaches. Ann Clin Biochem 2018;1:000456321876639.

22. Nieuwenburg SAV, Vuik FER, Kruip M, et al. Effect of anticoagulants and NSAIDs on accuracy of faecal immunochemical tests (FITs) in colorectal cancer screening: a systematic review and meta-analysis. Gut 2018. doi: 10.1136/gutjnl-2018-316344. [Epub ahead of print: 5 Jun 2018].

23. Gies A, Cuk K, Schrotz-King P, et al. Direct comparison of diagnostic performance of 9 quantitative fecal immunochemical tests for colorectal cancer screening. Gastroenterology 2018;154:93-104.

24. Carroll MRR, Piggott C, Pearson S. Evaluation of quantitative faecal immunochemical tests for haemoglobin. Guildford, UK: Guildford Medical Device Evaluation Centre (GMEC), 2013.

25. Piggott C, John C, Bruce $H$, et al. Does the mass of sample loaded affect faecal haemoglobin concentration using the faecal immunochemical test? Ann Clin Biochem 2018;1:000456321877870.

26. Rapi S, Berardi M, Cellai F, et al. Effects of fecal sampling on preanalytical and analytical phases in quantitative fecal immunochemical tests for hemoglobin. Int J Biol Markers 2017;32:261-6.

27. Carroll MR, John C, Mantio D, et al. An assessment of the effect of haemoglobin variants on detection by faecal immunochemical tests. Ann Clin Biochem 2018:1:000456321877871.

28. Kinns H, Pitkin S, Housley D, et al. Internal quality control: best practice. J Clin Pathol 2013;66:1027-32.

29. Fraser CG, Allison JE, Halloran SP, et al. A proposal to standardize reporting units for fecal immunochemical tests for hemoglobin. J Natl Cancer Inst 2012:104:810-4.

30. Clinical and Laboratory Standards Institute. Evaluation of detection capability for clinical laboratory measurement procedures. 2nd edn. Wayne, PA, USA: CLSI, 2012.

31. Fraser CG, Benton SC. Detection capability of quantitative faecal immunochemical tests for haemoglobin (FIT) and reporting of low faecal haemoglobin concentrations. Clin Chem Lab Med 2018:2018. 